\title{
Review
}

\section{Lentiviral Vectors and Antiretroviral Intrinsic Immunity}

\author{
BASTIEN MANGEAT and DIDIER TRONO
}

\begin{abstract}
Multicellular organisms have evolved under relentless attacks from pathogens, and as a consequence have spiked their genomes with numerous genes that serve to thwart these threats, notably through the building of the innate and adaptive arms of the immune system. The innate immune system is by far the most ancient, being found as widely as in plants and Drosophila, while adaptive immunity arose with the emergence of cartilaginous fishes. Innate immunity enters rapidly into the game during the course of an infection and generally involves the recognition by specific cellular receptors of common pathogen-associated patterns to elicit broad defensive responses, mediated in humans by interferons, macrophages, and natural killer cells, amongst others. When innate immunity fails to eradicate the infection quickly, adaptive immune responses enter into play, to generate exquisitely specific defenses to virtually any pathogen, thanks to a quasi-infinite repertoire of nonself receptors and effectors. A specific form of innate immunity, coined "intrinsic immunity," completes this protection by providing a constant, always-on, line of defense, generally through intracellular obstacles to the replication of pathogens. This component of the immune system has gained much attention as it was discovered that it is a cornerstone of the resistance of mammals against retroviruses. One of these newly discovered intracellular molecular weapons, the APOBEC family of proteins, is active against several classes of retroelements. We present here the current state of knowledge on this rapidly evolving field and discuss implications for gene therapy.
\end{abstract}

\section{INTRINSIC IMMUNITY THROUGH "LETHAL EDITING"}

$\mathbf{E}$ DITING IS A PROCESS by which genetic information is modified through the enzymatic modification of nucleotides in RNA or DNA. The discovery that editing enzymes were crucial components of intrinsic immunity stemmed from the elucidation of one of the most long-lasting puzzle of human immunodeficiency virus (HIV) research, namely the role of the HIV Vif protein. After the cloning of the HIV-1 genome more than 20 years ago, the functions of most of its nine genes were rapidly identified. However, the role of Vif remained elusive, a particularly vexing situation considering its absolute requirement for HIV-1 replication in vivo. It was rapidly recognized that some "restrictive" cells, including macrophages and primary $\mathrm{T}$ cells, could not support replication of HIV-1 deleted for the Vif gene, in contrast with many other cells, defined as "permissive" (Gabuzda et al., 1992; von Schwedler et al., 1993). Heterokaryon-based studies revealed that restrictive cells contained an antiviral factor, the presence of which in producer cells, rather than in target cells, was determinant (Madani and Kabat, 1998; Simon et al., 1998). Through subtractive hybridization studies between restrictive and permissive cells, Malim and coworkers identified APOBEC3G (apolipoprotein B mRNA-editing enzyme, catalytic polypeptide-like 3G), a member of a large cytidine deaminase family, as being the crucial antiviral factor (Sheehy et al., 2002). When present in the producer cell, and in the absence of Vif, APOBEC3G is efficiently packaged into budding virions, through a mechanism that remains to be fully defined but that involves its binding to the viral Gag protein (Cen et al., 2004; Svarovskaia et al., 2004). The enzyme then stays associated with the reverse transcription complex in the target cell, where it heavily deaminates cytidine residues in the nascent single-stranded DNA reverse transcripts

School of Life Sciences and "Frontiers in Genetics" National Center for Competence in Research, Swiss Federal Institute of Technology, 1015 Lausanne, Switzerland. 
(Harris et al., 2003; Lecossier et al., 2003; Mangeat et al., 2003; Zhang et al., 2003). This is thought to have two consequences: the uracil-containing DNA molecules are degraded, probably through the intervention of DNA repair enzymes, while those viral genomes that escape degradation are profoundly mutated and fail to produce functional proteins, thereby halting further viral spread (Fig. 1A). Facing this highly efficient attack in the host cells, lentiviruses evolved a counterstrike. The vif gene serves this role by connecting APOBEC3G to a Cul5-containing E3 ubiquitin ligase complex (Yu et al., 2003). This induces the proteasome-mediated degradation of the cellular enzyme, thus efficiently preventing its virion encapsidation (Mariani et al., 2003; Sheehy et al., 2003).

Adding to its importance as an antiviral defense, APOBEC3G antiviral activity is not limited to $\Delta$ Vif HIV-1 but extends to a broad range of wild-type lentiviruses naturally infecting nonhuman hosts, such as simian immunodeficiency virus (SIV) and equine infectious anemia virus (EIAV), and to more distantly related retroviruses such as murine leukemia virus (MLV) (Mangeat et al., 2003) and foamy viruses (Lochelt et al., 2005) (Table 1). APOBEC3G-mediated DNA hypermutation therefore constitutes a barrier to the cross-species transmission of a large array of retroviral pathogens.

The mode of action of the APOBEC family on singlestranded reverse transcripts supported the notion that certain of its members could also exert a detrimental action on the replication of other types of retroelements. Hepadnaviruses, such as hepatitis B virus (HBV), were ideal candidates because they also replicate their genome through the reverse transcription of a pregenomic RNA into a double-stranded DNA molecule, a process that takes place entirely in an intracytoplasmic viral core before virion release. As suspected, APOBEC3G does affect $\mathrm{HBV}$ replication through the inhibition of the accumulation of reverse transcripts, although the deamination of HBV DNA appears not to be essential (Turelli et al., 2004a) (Fig. 1B). Indeed, catalytically dead mutants of APOBEC $3 \mathrm{G}$ affected HBV replication as efficiently as the wild-type enzyme. The precise antiviral mechanism at play against HBV still awaits further investigation, although an inhibition of packaging of the pregenomic RNA or destabilization of the reverse transcription complex are possible explanations (Turelli et al., 2004a).

\section{THE APOBEC FAMILY: AT THE EDGE OF INNATE AND ADAPTIVE IMMUNITY}

APOBEC3G is part of a cluster of related genes located on human chromosome 22, which comprises APOBEC3A to $\mathrm{APOBEC} 3 \mathrm{H}$ and is specifically found in primates (Jarmuz et $a l .$, 2002) (Table 1). Other mammals such as mice or pigs contain only the likely precursor of these genes, namely APOBEC3. None of the putative nonantiviral physiological functions of the APOBEC3 proteins is currently understood, but several of them, in addition to APOBEC3G, have antiviral potential. APOBEC3F, for instance, exhibits strong anti-HIV activity, countered by Vif (Liddament et al., 2004; Wiegand et al., 2004; Zheng et al., 2004). Accordingly, the pattern of mutations found in proviruses isolated from AIDS patients matches not only the APOBEC3G consensus target, but also the slightly different pattern characteristic of APOBEC3F. In addition to the chro- mosome 22 APOBEC3 cluster, the family also contains three other genes, which are shared by all mammals and are expressed in a narrowly tissue-specific manner: APOBEC1, AID, and APOBEC2 (Table 1). Whereas the function of APOBEC2 is unknown, APOBEC1 is known to be involved in the regulation of lipid metabolism in small intestine enterocytes. There, it edits a specific cytidine residue of the apolipoprotein B (ApoB) mRNA into uridine, thus creating a stop codon that results in the translation of a shorter ApoB protein (Wedekind et al., 2003). Although this human enzyme is inactive against retroviruses, a report (Bishop et al., 2004) considerably broadens the potential spectrum of APOBEC targets, with the demonstration that rat APOBEC1 can deaminate not only a retroviral single-stranded DNA but also its genomic RNA precursor. The other well-known member of the family is AID, a pillar of adaptive immunity as it is the factor responsible for both class switch recombination (CSR) and somatic hypermutation (SHM) of immunoglobulin genes, two steps essential for antibody diversification (Luo et al., 2004). No antiviral activity has been attributed so far to AID.

The APOBEC family thus comprises members crucial for the adaptive and innate arms of immunity. This raises the intriguing possibility that a common APOBEC precursor, involved in primitive intrinsic host protection against retroelements, gave rise to a protein involved in antibody diversification by refocusing its action from the pathogens DNA to the cellular immunoglobulin genes. This is supported by the finding that AID, like APOBEC3 proteins, deaminates single-stranded, rather than double-stranded, DNA targets (Chaudhuri et al., 2003). The primitive pathogenic targets of the ancestral APOBEC could have been retrotransposons, the ancestors of modern retroviruses. These endogenous retroelements, which comprise long interspersed nuclear elements (LINEs), short interspersed nuclear elements (SINEs), and long terminal repeat (LTR) retrotransposons and account for a massive total of about $40 \%$ of the human genome (International Human Genome Sequencing Consortium, 2001), could have constituted a danger for host genome integrity through their random, and potentially mutagenic, transposition. Although the genomes of endogenous retroelements are usually silenced through methylation (Boeke and Stoye, 1997), they nevertheless are thought to retrotranspose in germ cells and during the genomic demethylation window that occurs in embryos at the preimplantation stage of development. In face of this potential threat, it is striking that several APOBEC3 proteins are highly active in vitro against diverse murine and even yeast retrotransposons and that the mouse genome contains a high number of retrotransposon proviruses that bear the scars of an APOBEC-mediated deamination (Dutko et al., 2005; Esnault et al., 2005). The reported expression of APOBEC3G and APOBEC3C in human germ cells could therefore be related to such protective action (Neuberger et al., 2003). Furthermore, the fact that only LINEs and SINEs are still active nowadays in the human genome is in good accordance with the lack of susceptibility of at least some LINEs to APOBEC3G action (Turelli et al., 2004b; Esnault et al., 2005).

The expansion of the APOBEC3 group might have subsequently provided the host cells with a broader range of antiviral mechanisms fighting different types of constantly diversifying retroelements, including exogenous retroviruses and hepad- 
A

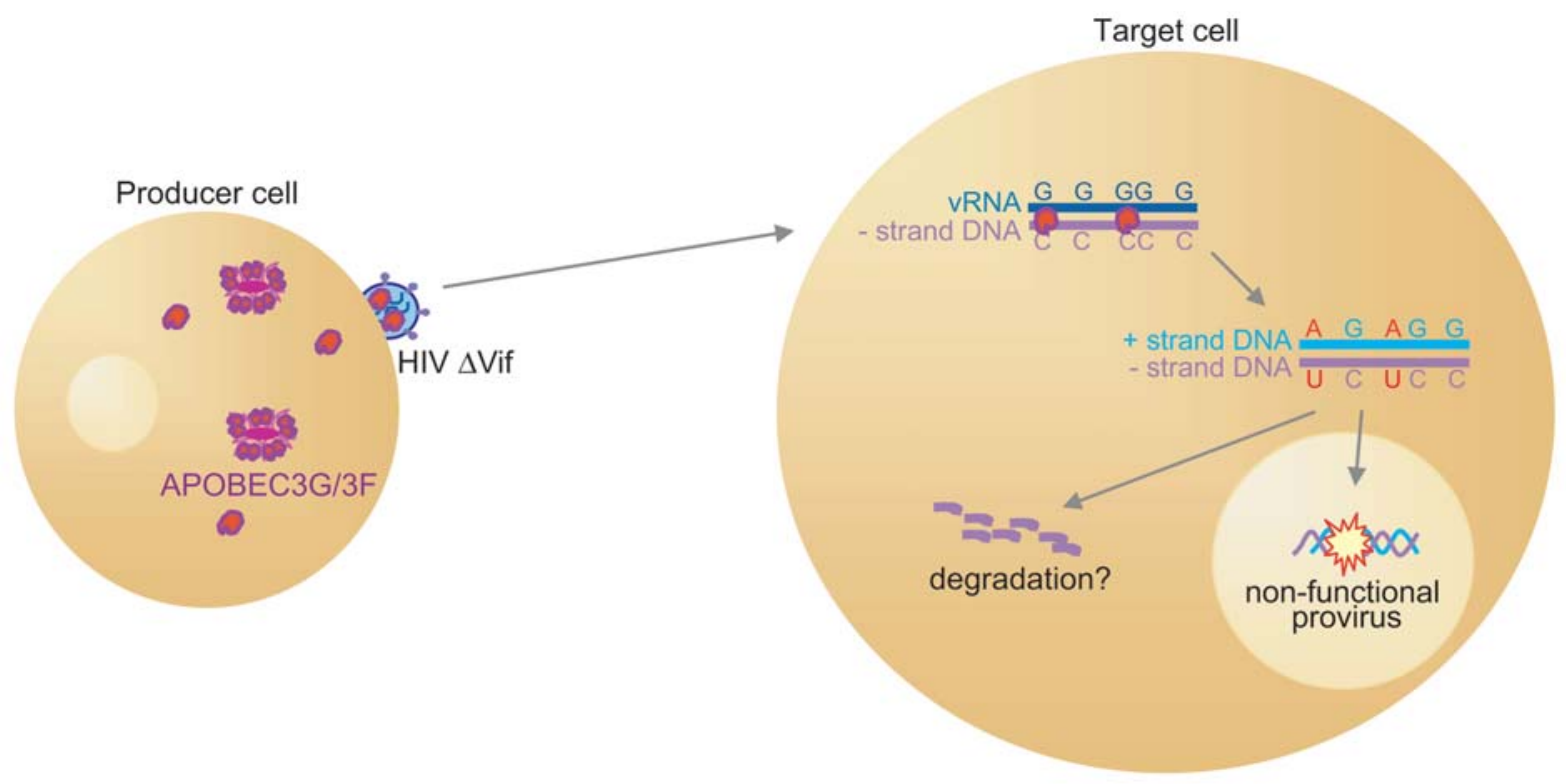

B

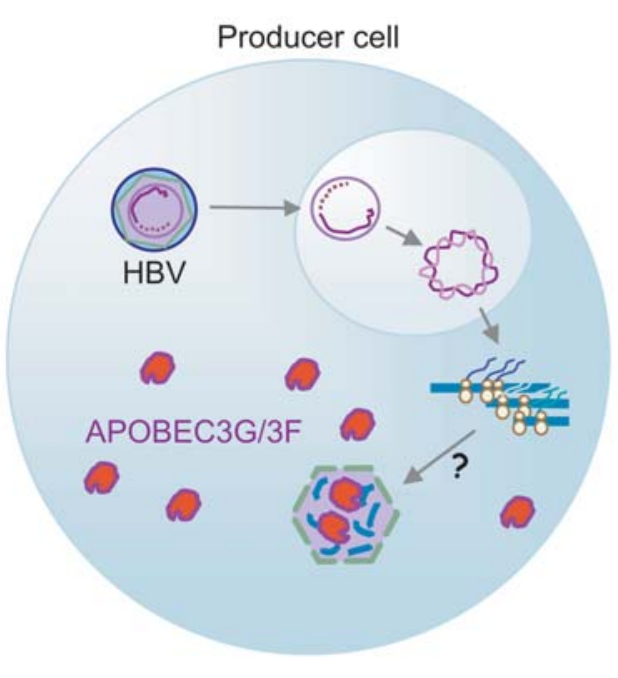

C

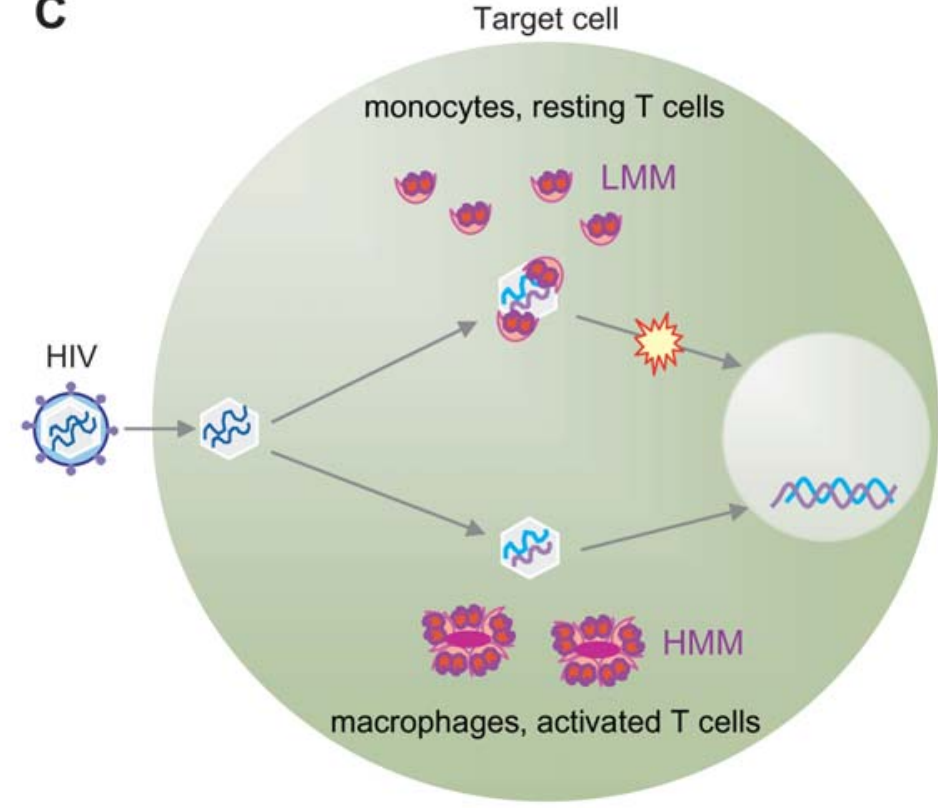

FIG. 1. The APOBEC family of proteins exerts its antiviral activity through several mechanisms. (A) APOBEC3G and APOBEC3F are encapsidated into budding HIV-1 virions. They then stay associated with the reverse transcription complex in the target cells, where they deaminate the viral DNA genome, halting further viral replication. (B) After the infection of a cell by HBV, the partially double-stranded DNA viral genome is transported into the nucleus. Its subsequent repair by cellular enzyme leads to the expression of viral proteins. These govern the packaging of a pregenomic RNA into cytoplasmic cores and its reverse transcription. The presence of APOBEC $3 \mathrm{G}$ or APOBEC $3 \mathrm{~F}$ in these cells results in a marked drop in HBV reverse transcript accumulation, probably through the inhibition of assembly or destabilization of viral cores. (C) After entering resting CD4 ${ }^{+}$ T lymphocytes or monocytes, HIV-1 is blocked by APOBEC3G, which associates with a low molecular mass (LMM) complex in these cells. On cellular activation, APOBEC3G relocalizes into a high molecular mass (HMM) complex, which relieves the restriction.

naviruses. Accordingly, APOBEC3C, which is inactive against HIV, has potent anti-SIV activity (Yu et al., 2004). In addition, the fact that several members of the family underwent strong positive selection, with an accelerated accumulation of non- synonymous changes during their evolution, strongly suggests that these proteins were involved in molecular conflicts, that is, served as defense possibly against different types of retroviruses (Sawyer et al., 2004; Zhang and Webb, 2004). 
Table 1. Characteristics of Human and Rodent APOBEC Family of Cytidine Deaminases ${ }^{a}$

\begin{tabular}{|c|c|c|c|c|c|}
\hline & \multicolumn{4}{|c|}{ Antiviral activity } & \multirow{2}{*}{$\begin{array}{l}H I V-1 \text { Vif } \\
\text { sensitivity }\end{array}$} \\
\hline & $H I V-1$ & $S I V$ & $M L V$ & $H B V$ & \\
\hline \multicolumn{6}{|c|}{ Human } \\
\hline APOBEC1 & - & ND & - & ND & ND \\
\hline APOBEC2 & - & - & ND & ND & ND \\
\hline AID & - & - & - & ND & ND \\
\hline APOBEC $3 \mathrm{~A}$ & - & ND & - & ND & ND \\
\hline APOBEC $3 B$ & $+1-$ & + & $+1-$ & ND & - \\
\hline APOBEC3C & - & + & - & ND & + \\
\hline APOBEC3D & ND & ND & ND & ND & ND \\
\hline APOBEC3E & ND & ND & ND & ND & ND \\
\hline APOBEC3DE & ND & ND & ND & ND & ND \\
\hline APOBEC3F & + & + & - & + & $+1-$ \\
\hline APOBEC3G & + & + & + & + & + \\
\hline АРОВЕС $3 \mathrm{H}$ & ND & ND & $\mathrm{ND}$ & ND & ND \\
\hline \multicolumn{6}{|c|}{ Rodent } \\
\hline APOBEC1 & $\begin{array}{c}\text { rat: }+ \\
\text { mouse: - }\end{array}$ & ND & - & ND & - \\
\hline APOBEC2 & - & ND & ND & ND & ND \\
\hline AID & ND & ND & ND & ND & ND \\
\hline APOBEC 3 & + & + & - & ND & - \\
\hline
\end{tabular}

Abbreviation: ND, not determined.

aThe antiviral activity against different retroelements and the sensitivity to HIV-1 Vif are listed for each enzyme.

\section{THE COUNTERSTRIKE OF ASSAULTED VIRUSES}

The potent antiretroviral mechanism of the APOBEC enzymes forced susceptible pathogens to develop adequate escapes. As stated previously, the lentiviral Vif proteins protect their cognate genomes by provoking the clearance of APOBEC3G from the viral producer cells. Yet, and in contrast to the broad action of APOBEC3G, the viral protection conferred by Vif appears to be highly species specific. Indeed, HIV-1 Vif protein can target the APOBEC3G protein from human (huAPOBEC3G) and chimpanzee, but not the one from macaques or African green monkeys (agmAPOBEC3G). Similarly, the Vif gene of SIVagm, a widespread simian virus, is unable to counteract huAPOBEC3G (Mariani et al., 2003). Interestingly, the speciesspecific sensitivity of APOBEC $3 \mathrm{G}$ to Vif action is governed by a single amino acid at position 128 of APOBEC3G (Bogerd et al., 2004; Mangeat et al., 2004; Schrofelbauer et al., 2004). Indeed, HIV-1 Vif acts by binding huAPOBEC3G, an event blocked when aspartic acid at position 128 of huAPOBEC $3 \mathrm{G}$ is replaced by the corresponding lysine found in agmAPOBEC3G. Moreover, this modified huAPOBEC $3 \mathrm{G}$ acquires sensitivity to the action of SIVagm Vif, and thus behaves just as agmAPOBEC3G does.

Two important implications stem from this species-specific action of Vif. First, the AIDS epidemics could arise only because SIVcpz and SIVmac, the viruses at the origin of HIV-1 and HIV-2, respectively, have vif genes that were able to counteract huAPOBEC3G action. Second, a putative animal model permitting HIV replication must possess an APOBEC3G that is susceptible to HIV-1 Vif.
Although the vif gene of lentiviruses efficiently fends off the APOBEC attack, other retroviruses have evolved other escape mechanisms. One example is the bet gene of foamy viruses (Lochelt et al., 2005). In addition, MLV, which replicates in murine APOBEC3-positive cells but does not have either a vif or a bet gene, survives because its Gag protein fails to be recognized by the murine deaminase, which is therefore excluded from released virions (Kobayashi et al., 2004; Doehle et al., 2005). Why MLV evolved such a simple escape mechanism while HIV and foamy viruses had to develop brand new genes to achieve the same goal is an intriguing question. The lentivirus EIAV, which is also devoid of a known counterstrike protein but is sensitive to human APOBEC3G, might also have developed its own mechanism to escape the action of the equine ortholog of APOBEC3. This might involve its selective replication in cells devoid of this enzyme, the presence in one of its proteins of a hidden Vif-like activity, or a twist that renders its Gag protein invisible to the deaminase.

\section{IMPLICATIONS FOR LENTIVECTOR PRODUCTION}

The presence in human cells of such a powerful antiretroviral mechanism has important implications for gene therapy. Indeed, even minute amounts of a harmful APOBEC protein in producer cells will undermine the successful use of retroviruses as a gene delivery system, through a decrease in virion infectivity and an increase of the proportion of defective particles, which favors the appearance of toxic effects and immune responses directed against virion components. Laboratories work- 
ing with specific vector systems, such as EIAV or SIV, would benefit from defining the susceptibility of their vectors to all APOBEC family members and from choosing producer cells devoid of any traces of relevant APOBEC activity.

In addition, some transgenes might activate the expression of a detrimental APOBEC in producer cells. The expression of APOBEC family members should therefore be checked when the production of high-titer vectors is proving difficult. Likewise, when a transgene fails to be expressed or to demonstrate correct effector functions despite adequate proviral DNA titer, the polymerase chain reaction (PCR) amplification of the proviruses in target cells might reveal the presence of APOBECinduced mutations potentially altering either promoter or coding sequences.

It would thus be beneficial to obtain a packaging system insensitive to human APOBEC inhibition, even in the absence of the viral vif gene. In this respect, much might be learned from the escape mechanisms of different retroviruses.

\section{APOBEC3G IN TARGET CELLS CAN ALSO BLOCK LENTIVECTOR TRANSDUCTION}

Resting $\mathrm{CD}^{+}{ }^{+}$cells are notorious for being resistant to HIV infection or lentivector transduction, imposing a block at an early postentry stage of viral replication (Zack et al., 1990; Korin and Zack, 1998; Ducrey-Rundquist et al., 2002). Because these cells constitute the vast majority of $\mathrm{T}$ cells in vivo, their targeting for lentivector-mediated gene treatment of immunodeficiencies or other diseases is complex. Light was shed on this problem when it was discovered that APOBEC $3 \mathrm{G}$ not only is the antiviral factor counteracted by Vif in producer cells, but is also responsible for this target cell resistance (Chiu et al., 2005) (Fig. 1C). In resting T cells and monocytes, APOBEC3G resides in a low molecular mass (LMM) complex as opposed to the high molecular mass (HMM) complex in which it is found in activated $\mathrm{T}$ cells, macrophages, and other cells. This LMM APOBEC 3G is responsible for the replication block through an undefined mechanism, which probably involves recruitment of the deaminase to the reverse transcription complex. Activating the $\mathrm{CD}^{+}{ }^{+} \mathrm{T}$ cells relocalizes APOBEC3G into the high molecular mass (HMM) complex and relieves the restriction. The design of putative HIV vectors capable of escaping this blockade will require the fine deciphering of its mechanism.

In addition to resting $\mathrm{T}$ cells, this complex might also be responsible for the low susceptibility of other cell types to transduction, be they of hematopoietic origin or otherwise. In this respect, it could be envisioned that murine APOBEC3, which is expressed in mouse gonads, could account for the block that murine oocytes impose to lentivectors during their first divisions (our unpublished data). Finally, it will be crucial to determine whether other APOBEC family members also are endowed with the ability to mediate such a mode of resistance.

\section{CYTIDINE DEAMINASE AS A GENE-BASED ANTIVIRAL THERAPY}

On the basis of the strong antiviral activity of APOBEC family members, it is tempting to propose their use for the gene therapy of several infectious diseases. Two potential approaches can be envisioned for the use of APOBEC3G as a tool to fight HIV-1 infection. These would take advantage of the antiviral action mediated by the APOBEC $3 \mathrm{G}$ residing in either the producer or the target cells.

\section{Producer cells}

The ex vivo transduction of patients' hematopoietic stem cells (HSCs) with a Vif-resistant APOBEC3G, followed by their reinjection, would provide the host with a pool of lymphocytes incapable of sustaining productive HIV replication. Although this approach will probably be efficient only if a large percentage of blood cells can be reconstituted by the transgenic precursors, it is hoped that this would slow down the infection and permit the amount of antiviral drugs administered to the patient to be reduced. The safety of this approach will nevertheless need to be carefully investigated. The alteration applied to human APOBEC3G to render it Vif resistant might indeed alter putative regulatory circuitries, potentially leading to random mutagenic deamination of the host genome. Arguing against this possibility, however, are African green monkeys that possess an HIV Vif-resistant APOBEC3G but are not especially prone to leukemia, raising hopes that the same will hold true in humans. In any case, using the HIV-1 LTR to drive the expression of the Vif-resistant APOBEC3G could solve the problem. In this situation, the potentially harmful enzyme would indeed be expressed only in infected cells, and only during the few days during which they can survive with the virus, thus minimizing exposure to dysregulated genomic deamination.

Alternatively, lentivectors expressing a small hairpin RNA (shRNA) against Cul5 under the control of the HIV-1 LTR might be introduced into HSCs. The advantage of this approach over conventional drug-based treatments is that viral resistances would certainly occur less frequently, because Vif would have to evolve a new mechanism of action.

\section{Target cells}

The transduction of blood precursors with a putative mutant of APOBEC $3 \mathrm{G}$ constitutively residing in the LMM complex, even upon T cell stimulation, would provide the cells with two APOBEC 3G pools: the endogenous APOBEC 3G, which would perform its putative physiological role, and the "LMM-resident" transgenic APOBEC $3 \mathrm{G}$, which would fight incoming viruses. Interestingly, the earliness of the step of infection at which the viruses would be blocked, before integration into the host genome, would hopefully allow for the preservation of enough uninfected T cells to maintain an effective immune system. Although this approach might prove dangerous if the LMM form of APOBEC $3 \mathrm{G}$ is more prone to induce deleterious nucleotide deaminations in the host genome in dividing cells than its HMM counterpart (Chiu et al., 2005). The problem could be tackled if a catalytically inactive APOBEC $3 \mathrm{G}$ could also mediate the block, a likely possibility because hypermutations do not seem to be involved in the restriction. Along the same line of thought, drugs locking APOBEC3G in the LMM complex could also induce an early intracellular resistance to retroviral infections, although they would also probably impede the putative physiological function of the enzyme.

To gain insights into the efficiency and safety of these different approaches, the variously modified APOBEC3Gs could be introduced into mice reconstituted with a human immune 
system (Traggiai et al., 2004), provided these prove able to sustain a productive HIV-1 infection.

These novel gene therapy approaches could stand as valuable alternatives, or supports, to current drug treatments, the efficiency of which is steadily ebbing as a result of resistance in a growing percentage of patients. It is therefore not surprising that the first human lentivector gene therapy trial, which is currently being evaluated in phase I clinical trials, is aimed at treating human HIV infection through the introduction into T cells of an HIV resistance gene (Humeau et al., 2004; Lu et al., 2004).

\section{APOBEC FAMILY AS AN ANTI-HBV THERAPY}

The chronic hepatitis resulting from HBV infection can be treated with lamivudine or interferon- $\alpha$ (IFN- $\alpha)$. However, resistant HBV clones often emerge during long-term lamivudine treatment, and IFN treatment is associated with severe side effects such as autoimmune disease and bone marrow reduction (Hollinger and Liang, 2001). Therefore, great efforts are being made to develop better treatments against this major cause of mortality and morbidity, amongst which gene therapy holds some promise (Xu et al., 2003).

Human hepatocytes do not express APOBEC3G or APOBEC3F, at least under normal circumstances. It can thus be envisioned that patients' liver may be rendered resistant to HBV through the adeno-associated viral or lentiviral vectormediated delivery of APOBEC $3 \mathrm{G}$ into their hepatocytes. Owing to the independence of APOBEC $3 \mathrm{G}$ anti-HBV activity from deamination, hepatocytes expressing a catalytically inactive form of the enzyme would be endowed with a safe mechanism to fight the infection.

The "APOBEC3G gene therapy" approach is interesting because it might actually recapitulate a bona fide defense occurring in hepatocytes in certain situations in vivo. It has, for instance, been shown that HBV DNA is cleared from infected hepatocytes during acute infection through an unidentified intracellular mechanism (Guidotti et al., 1999). The fact that this protection seems induced by cytokines in vivo and by IFN in culture raises the possibility that an APOBEC family member might be upregulated in hepatocytes subsequent to these treatments to mediate HBV clearance. In this respect, it is worth noting that APOBEC3A is much upregulated in peripheral blood mononuclear cells (PBMCs) on IFN- $\alpha$ treatment (Taylor et al., 2004). In addition, the occasional G-to-A hypermutant $\mathrm{HBV}$ clones that have been detected in patients might constitute by-products of the anti-HBV action of an APOBEC family member.

It will be interesting to determine precisely whether other APOBECs are active against HBV, and whether any of them is constitutively expressed in hepatocytes or can be upregulated by inflammation, HBV infection, or pharmacological treatment.

\section{APOBEC FAMILY INVOLVEMENT IN CANCERS}

One of the most frequent genomic point mutations, the Cto- $T$ transition, arises through the deamination of cytidines into uridines (Lodish, 2003). Moreover, about 23\% of mutations in hereditary human diseases and $24 \%$ of mutations in p53 in human cancers are G-to-A transitions (Waters and Swann, 2000). It has also been noted that the base pair context of mutation in different oncogenes or tumor suppressors vary between cell types (Beale et al., 2004). It can thus be argued that what are currently considered to be "spontaneous mutations" could actually reflect the action of upregulated, dysregulated, or ectopically expressed APOBEC enzymes. Accordingly, the expression of APOBEC family members has been demonstrated in many cancerous cells (Jarmuz et al., 2002), certain leukemic B cells show hallmarks of uncontrolled AID-mediated hypermutation in oncogenes, and the APC gene in colorectal tumors harbors a pattern of mutations compatible with APOBEC1 editing (Beale et al., 2004). Finally, APOBEC1 transgenic expression in liver leads to cancer in mice (Yamanaka et al., 1995). Within the context of a precancerous tissue, cytidine deamination could therefore provide a continuous source of potentially oncogenic mutations, in a stepwise progression toward metastasis development. Therefore, whether the presence of an upregulated APOBEC could serve as a prognostic tool for the aggressiveness of the tumor is worth further investigation.

But most importantly, the inherent danger residing in the ectopic delivery of these deaminases in cells might preclude their future use as safe molecular tools for gene-based therapies.

\section{PERSPECTIVES}

We have focused this review on the action of the APOBEC family against retroelements. However, other viral families might be affected, as well as their related gene delivery systems. For instance, vaccinia vectors, which replicate their DNA in the cytoplasm, where APOBEC $3 \mathrm{G}$ is located, can efficiently transduce monocytes, B cells, and natural killer cells, but not T lymphocytes (Sanchez-Puig et al., 2004). The perspective that APOBEC3G might be responsible for this blockade paves the way for further provocative and stimulating research.

Although APOBEC3G and several other APOBEC family members provide antiretroviral protection, it is probable that mammalian cells contain many other unrecognized intrinsic antiviral effectors. The newly discovered Trim5-mediated block to retroviral infection constitutes one such example. Identifying these factors and unraveling their mode of action will certainly lead to advances in gene therapy and, in more general terms, in molecular virology.

\section{ACKNOWLEDGMENTS}

We thank Priscilla Turelli for help in the redaction of this manuscript, and apologize to those investigators whose work could not be cited because of space limitations. Research in our laboratory is supported by the Swiss National Science Foundation, the European Union, and the Institut Clayton de la Recherche.

\section{REFERENCES}

BEALE, R.C., PETERSEN-MAHRT, S.K., WATT, I.N., HARRIS, R.S., RADA, C., and NEUBERGER, M.S. (2004). Comparison of 
the differential context-dependence of DNA deamination by APOBEC enzymes: Correlation with mutation spectra in vivo. J. Mol. Biol. 337, 585-596.

BISHOP, K.N., HOLMES, R.K., SHEEHY, A.M., and MALIM, M.H. (2004). APOBEC-mediated editing of viral RNA. Science 305, 645

BOEKE, J.D., and STOYE, J.P. (1997). Retrotransposons, endogenous retroviruses, and the evolution of retroelements. In Retroviruses. J.M. Coffin, S.H. Hughes, and H. Varmus, eds. (Cold Spring Harbor Laboratory Press, Plainview, NY) pp. 343-435.

BOGERD, H.P., DOEHLE, B.P., WIEGAND, H.L., and CULLEN, B.R. (2004). A single amino acid difference in the host APOBEC3G protein controls the primate species specificity of HIV type 1 virion infectivity factor. Proc. Natl. Acad. Sci. U.S.A. 101, 3770-3774.

CEN, S., GUO, F., NIU, M., SAADATMAND, J., DEFLASSIEUX, J., and KLEIMAN, L. (2004). The interaction between HIV-1 Gag and APOBEC3G. J. Biol. Chem. 279, 33177-33184.

CHAUDHURI, J., TIAN, M., KHUONG, C., CHUA, K., PINAUD, E., and ALT, F.W. (2003). Transcription-targeted DNA deamination by the AID antibody diversification enzyme. Nature 422, 726-730.

CHIU, Y.L., SOROS, V.B., KREISBERG, J.F., STOPAK, K., YONEMOTO, W., and GREENE, W.C. (2005). Cellular APOBEC3G restricts HIV-1 infection in resting $\mathrm{CD}^{+}{ }^{+} \mathrm{T}$ cells. Nature 435, 108 114.

DOEHLE, B.P., SCHAFER, A., WIEGAND, H.L., BOGERD, H.P., and CULLEN, B.R. (2005). Differential sensitivity of murine leukemia virus to APOBEC3-mediated inhibition is governed by virion exclusion. J. Virol. 79, 8201-8207.

DUCREY-RUNDQUIST, O., GUYADER, M., and TRONO, D. (2002). Modalities of interleukin-7-induced human immunodeficiency virus permissiveness in quiescent T lymphocytes. J. Virol. 76, 9103-9111.

DUTKO, J.A., SCHAFER, A., KENNY, A.E., CULLEN, B.R., and CURCIO, M.J. (2005). Inhibition of a yeast LTR retrotransposon by human APOBEC3 cytidine deaminases. Curr. Biol. 15, 661-666.

ESNAULT, C., HEIDMANN, O., DELEBECQUE, F., DEWANNIEUX, M., RIBET, D., HANCE, A.J., HEIDMANN, T., and SCHWARTZ, O. (2005). APOBEC3G cytidine deaminase inhibits retrotransposition of endogenous retroviruses. Nature 433, 430-433.

GABUZDA, D.H., LAWRENCE, K., LANGHOFF, E., TERWILLIGER, E., DORFMAN, T., HASELTINE, W.A., and SODROSKI, J. (1992). Role of Vif in replication of human immunodeficiency virus type 1 in $\mathrm{CD}^{+} \mathrm{T}$ lymphocytes. J. Virol. 66, 6489-6495.

GUIDOTTI, L.G., ROCHFORD, R., CHUNG, J., SHAPIRO, M., PURCELL, R., and CHISARI, F.V. (1999). Viral clearance without destruction of infected cells during acute HBV infection. Science 284, 825-829.

HARRIS, R.S., BISHOP, K.N., SHEEHY, A.M., CRAIG, H.M., PETERSEN-MAHRT, S.K., WATT, I.N., NEUBERGER, M.S., and MALIM, M.H. (2003). DNA deamination mediates innate immunity to retroviral infection. Cell 113, 803-809.

HOLLINGER, F.B., and LIANG, T.J. (2001). Hepatitis B virus. In Fields' Virology. B.N. Fields, D.M. Knipe, P.M. Howley, and D.E. Griffin, eds. (Lippincott Williams \& Wilkins, Philadelphia, PA) Chapter 87, pp. 2809-2818.

HUMEAU, L.M., BINDER, G.K., LU, X., SLEPUSHKIN, V., MERLING, R., ECHEAGARAY, P., PEREIRA, M., SLEPUSHKINA, T., BARNETT, S., DROPULIC, L.K., CARROLL, R., LEVINE, B.L., JUNE, C.H., and DROPULIC, B. (2004). Efficient lentiviral vectormediated control of HIV-1 replication in CD4 lymphocytes from diverse $\mathrm{HIV}^{+}$infected patients grouped according to CD4 count and viral load. Mol. Ther. 9, 902-913.

INTERNATIONAL HUMAN GENOME SEQUENCING CONSORTIUM (2001). Initial sequencing and analysis of the human genome. Nature 409, 860-921.

JARMUZ, A., CHESTER, A., BAYLISS, J., GISBOURNE, J., DUNHAM, I., SCOTT, J., and NAVARATNAM, N. (2002). An anthro- poid-specific locus of orphan $\mathrm{C}$ to U RNA-editing enzymes on chromosome 22. Genomics 79, 285-296.

KOBAYASHI, M., TAKAORI-KONDO, A., SHINDO, K., ABUDU, A., FUKUNAGA, K., and UCHIYAMA, T. (2004). APOBEC3G targets specific virus species. J. Virol. 78, 8238-8244.

KORIN, Y.D., and ZACK, J.A. (1998). Progression to the G1b phase of the cell cycle is required for completion of human immunodeficiency virus type 1 reverse transcription in T cells. J. Virol. 72, 3161-3168.

LECOSSIER, D., BOUCHONNET, F., CLAVEL, F., and HANCE, A.J. (2003). Hypermutation of HIV-1 DNA in the absence of the Vif protein. Science 300, 1112.

LIDDAMENT, M.T., BROWN, W.L., SCHUMACHER, A.J., and HARRIS, R.S. (2004). APOBEC3F properties and hypermutation preferences indicate activity against HIV-1 in vivo. Curr. Biol. 14, 1385-1391

LOCHELT, M., ROMEN, F., BASTONE, P., MUCKENFUSS, H., KIRCHNER, N., KIM, Y.B., TRUYEN, U., ROSLER, U., BATTENBERG, M., SAIB, A., FLORY, E., CICHUTEK, K., and MUNK, C. (2005). The antiretroviral activity of APOBEC3 is inhibited by the foamy virus accessory Bet protein. Proc. Natl. Acad. Sci. U.S.A. 102, 7982-7987.

LODISH, H.F. (2003). Cancer. In Molecular Cell Biology. H.F. Lodish, ed. (W.H. Freeman, New York) Chapter 23, pp. 935-973.

LU, X., YU, Q., BINDER, G.K., CHEN, Z., SLEPUSHKINA, T., ROSSI, J., and DROPULIC, B. (2004). Antisense-mediated inhibition of human immunodeficiency virus (HIV) replication by use of an HIV type 1-based vector results in severely attenuated mutants incapable of developing resistance. J. Virol. 78, 7079-7088.

LUO, Z., RONAI, D., and SCHARFF, M.D. (2004). The role of activation-induced cytidine deaminase in antibody diversification, immunodeficiency, and B-cell malignancies. J. Allergy Clin. Immunol. 114, 726-735; quiz, 736.

MADANI, N., and KABAT, D. (1998). An endogenous inhibitor of human immunodeficiency virus in human lymphocytes is overcome by the viral Vif protein. J. Virol. 72, 10251-10255.

MANGEAT, B., TURELLI, P., CARON, G., FRIEDLI, M., PERRIN, L., and TRONO, D. (2003). Broad antiretroviral defence by human APOBEC3G through lethal editing of nascent reverse transcripts. Nature 424, 99-103.

MANGEAT, B., TURELLI, P., LIAO, S., and TRONO, D. (2004). A single amino acid determinant governs the species-specific sensitivity of APOBEC3G to Vif action. J. Biol. Chem. 279, 14481-14483.

MARIANI, R., CHEN, D., SCHROFELBAUER, B., NAVARRO, F., KONIG, R., BOLLMAN, B., MUNK, C., NYMARK-MCMAHON, H., and LANDAU, N.R. (2003). Species-specific exclusion of APOBEC3G from HIV-1 virions by Vif. Cell 114, 21-31.

NEUBERGER, M.S., HARRIS, R.S., DI NOIA, J., and PETERSENMAHRT, S.K. (2003). Immunity through DNA deamination. Trends Biochem. Sci. 28, 305-312.

SANCHEZ-PUIG, J.M., SANCHEZ, L., ROY, G., and BLASCO, R. (2004). Susceptibility of different leukocyte cell types to vaccinia virus infection. Virol. J. 1, 10.

SAWYER, S.L., EMERMAN, M., and MALIK, H.S. (2004). Ancient adaptive evolution of the primate antiviral DNA-editing enzyme APOBEC3G. PLoS Biol. 2, E275.

SCHROFELBAUER, B., CHEN, D., and LANDAU, N.R. (2004). A single amino acid of APOBEC3G controls its species-specific interaction with virion infectivity factor (Vif). Proc. Natl. Acad. Sci. U.S.A. 101, 3927-3932.

SHEEHY, A.M., GADDIS, N.C., CHOI, J.D., and MALIM, M.H. (2002). Isolation of a human gene that inhibits HIV-1 infection and is suppressed by the viral Vif protein. Nature 418, 646-650.

SHEEHY, A.M., GADDIS, N.C., and MALIM, M.H. (2003). The antiretroviral enzyme APOBEC $3 \mathrm{G}$ is degraded by the proteasome in response to HIV-1 Vif. Nat. Med. 9, 1404-1407. 
SIMON, J.H., GADDIS, N.C., FOUCHIER, R.A., and MALIM, M.H. (1998). Evidence for a newly discovered cellular anti-HIV-1 phenotype. Nat. Med. 4, 1397-1400.

SVAROVSKAIA, E.S., XU, H., MBISA, J.L., BARR, R., GORELICK, R.J., ONO, A., FREED, E.O., HU, W.S., and PATHAK, V.K. (2004). Human apolipoprotein B mRNA-editing enzyme-catalytic polypeptide-like 3G (APOBEC3G) is incorporated into HIV-1 virions through interactions with viral and nonviral RNAs. J. Biol. Chem. 279, 35822-35828.

TAYLOR, M.W., GROSSE, W.M., SCHALEY, J.E., SANDA, C., WU, X., CHIEN, S.C., SMITH, F., WU, T.G., STEPHENS, M., FERRIS, M.W., MCCLINTICK, J.N., JEROME, R.E., and EDENBERG, H.J. (2004). Global effect of PEG-IFN- $\alpha$ and ribavirin on gene expression in PBMC in vitro. J. Interferon Cytokine Res. 24, 107-118.

TRAGGIAI, E., CHICHA, L., MAZZUCCHELLI, L., BRONZ, L., PIFFARETTI, J.C., LANZAVECCHIA, A., and MANZ, M.G. (2004). Development of a human adaptive immune system in cord blood cell-transplanted mice. Science 304, 104-107.

TURELLI, P., MANGEAT, B., JOST, S., VIANIN, S., and TRONO, D. (2004a). Inhibition of hepatitis B virus replication by APOBEC3G. Science 303, 1829.

TURELLI, P., VIANIN, S., and TRONO, D. (2004b). The innate antiretroviral factor APOBEC3G does not affect human LINE-1 retrotransposition in a cell culture assay. J. Biol. Chem. 279, 4337143373.

VON SCHWEDLER, U., SONG, J., AIKEN, C., and TRONO, D. (1993). Vif is crucial for human immunodeficiency virus type 1 proviral DNA synthesis in infected cells. J. Virol. 67, 4945-4955.

WATERS, T.R., and SWANN, P.F. (2000). Thymine-DNA glycosylase and $\mathrm{G}$ to $\mathrm{A}$ transition mutations at $\mathrm{CpG}$ sites. Mutat. Res. 462, 137-147.

WEDEKIND, J.E., DANCE, G.S., SOWDEN, M.P., and SMITH, H.C. (2003). Messenger RNA editing in mammals: New members of the APOBEC family seeking roles in the family business. Trends Genet. 19, 207-216.

WIEGAND, H.L., DOEHLE, B.P., BOGERD, H.P., and CULLEN, B.R. (2004). A second human antiretroviral factor, APOBEC3F, is suppressed by the HIV-1 and HIV-2 Vif proteins. EMBO J. 23, 2451-2458.

XU, R., CAI, K., ZHENG, D., MA, H., XU, S., and FAN, S.T. (2003). Molecular therapeutics of HBV. Curr. Gene Ther. 3, 341-355.
YAMANAKA, S., BALESTRA, M.E., FERRELL, L.D., FAN, J., ARNOLD, K.S., TAYLOR, S., TAYLOR, J.M., and INNERARITY, T.L. (1995). Apolipoprotein B mRNA-editing protein induces hepatocellular carcinoma and dysplasia in transgenic animals. Proc. Natl. Acad. Sci. U.S.A. 92, 8483-8487.

YU, Q., CHEN, D., KONIG, R., MARIANI, R., UNUTMAZ, D., and LANDAU, N.R. (2004). APOBEC3B and APOBEC3C are potent inhibitors of simian immunodeficiency virus replication. J. Biol. Chem. 279, 53379-53386.

YU, X., YU, Y., LIU, B., LUO, K., KONG, W., MAO, P., and YU, X.F. (2003). Induction of APOBEC3G ubiquitination and degradation by an HIV-1 Vif-Cul5-SCF complex. Science 302, 1056-1060. ZACK, J.A., ARRIGO, S.J., WEITSMAN, S.R., GO, A.S., HAISLIP, A., and CHEN, I.S. (1990). HIV-1 entry into quiescent primary lymphocytes: Molecular analysis reveals a labile, latent viral structure. Cell 61, 213-222.

ZHANG, H., YANG, B., POMERANTZ, R.J., ZHANG, C., ARUNACHALAM, S.C., and GAO, L. (2003). The cytidine deaminase CEM15 induces hypermutation in newly synthesized HIV-1 DNA. Nature 424, 94-98.

ZHANG, J., and WEBB, D.M. (2004). Rapid evolution of primate antiviral enzyme APOBEC3G. Hum. Mol. Genet. 13, 1785-1791.

ZHENG, Y.H., IRWIN, D., KUROSU, T., TOKUNAGA, K., SATA, T., and PETERLIN, B.M. (2004). Human APOBEC3F is another host factor that blocks human immunodeficiency virus type 1 replication. J. Virol. 78, 6073-6076.

\section{Address reprint requests to: Prof. Didier Trono School of Life Sciences Swiss Federal Institute of Technology EPFL SV-DO, AAB-Station 15 1015 Lausanne, Switzerland \\ E-mail: didier.trono@epfl.ch}

Received for publication June 23, 2005; accepted July 6, 2005.

Published online: July 18, 2005. 